\title{
Fuch's corneal dystrophy in a patient with mitochondrial DNA mutations
}

Roger L Albin

\begin{abstract}
A patient with Fuch's corneal dystrophy, sensorineural hearing loss, diabetes, cardiac conduction defects, ataxia, and hyperreflexia is described. Analysis of lymphocyte mitochondrial DNA showed missense mutations usually associated with Leber's hereditary optic neuropathy. The occurrence of Fuch's dystrophy in this patient and the biology of corneal endothelial cells suggest that mitochondrial defects could be the cause of Fuch's endothelial dystrophy.

( 7 Med Genet 1998;35:258-259)
\end{abstract}

Keywords: LHON; Leber's hereditary optic neuropathy

Mitochondrial genetic defects are associated with a broad range of organ system disorders. ${ }^{1}$ I recently encountered a patient with Fuch's corneal dystrophy and a mitochondrial disorder. While visual system disorders, notably retinopathy and optic neuropathy, are common in mitochondrial disorders, corneal dystrophy has not been reported previously.

\section{Case report}

The proband is a 48 year old woman evaluated because of a progressive decline in gait and the presence of involuntary movements. The patient's birth weight was 2948 g. Early growth and development were reportedly normal. She developed bilateral sensorineural hearing loss aged 12. At the age of 19, she had an acute neurological episode described by her spouse as "meningitis" associated with worsening of her hearing. Corneal abnormalities were noted in her late teens. When married at the age of 21 , she had normal speech, gait, and strength. Her ophthalmological care was provided at the Department of Ophthalmology of the University of Michigan and she was noted to have progressive corneal oedema and endothelial degeneration. She was subsequently diagnosed with Fuch's endothelial dystrophy and under-

Revised version accepted

for publication

8 September 1997 went bilateral keratoplasties. In her early 20 s, she developed progressive speech and gait difficulties over a period of several months. Neurological examination in 1974 disclosed dysarthria, normal power, slowed rapid and rhythmic alternating movements, mild upper extremity tremor, wide based, unsteady gait with inability to tandem walk, and lower extremity hyperreflexia. Her CSF protein was 69 with other CSF studies normal. Pneumoencephalography showed cerebral cortical atrophy with probable cerebellar atrophy. Skin biopsy was normal with negative staining for mucopolysaccharides. Amyloid staining of a colon biopsy was negative. No metachromatic granules were found in the urine. LE prep and anti-DNA were negative. Electromyography and nerve conductions were normal. Torch screen was normal, as were thyroid function tests. Biochemical evaluation for mucopolysaccharidoses and metachromatic leucodystrophy was normal. EKG showed complete right bundle branch block with anterior ventricular block. Glucose tolerance test was positive and oral hypoglycaemic agents were recommended.

Several of these problems progressed over the next 20 years. Her visual function worsened with the development of secondary glaucoma and her diabetes eventually required insulin therapy. Her neurological function deteriorated slowly with increasing gait impairment and impaired upper extremity function. She became deaf and required cochlear implantation.

Only an incomplete family history was obtained (fig 1). The subject's sister suffered from diabetes and a poorly described visual problem. The patient's brother had died suddenly at a relatively young age, had epilepsy, and had some form of extraocular muscle problem. Two of the sister's children had epilepsy but the brother's two children are reportedly normal.

She was re-examined in 1996. She was a short woman $(<1.5 \mathrm{~m})$ in no distress. Her mental status was normal. Cranial nerve examination showed small unreactive pupils, a right exotropia with full extraocular movements, and a mild right ptosis. She had moderate dysarthria. Muscle power was $5 / 5$ in all groups tested in all four extremities. Fingernose-finger testing showed mild dystaxia. Similar dystaxia was present in her legs. Rapid, rhythmic alternating movements were slowed and irregular. She was able to stand without assistance but had a widened base and impaired postural reflexes. Gait was ataxic with poor tandem gait. Mild choreic movements and occasional dystonic limb posturing were 
noted. Sensory examination showed normal pinprick and light touch sensation but diminished vibratory sensation in the feet. DTRs were $2+$ /symmetrical in the arms and $3+/$ symmetrical in the legs. Plantar responses were equivocal.

MRI showed markedly decreased brain volume and diffuse subcortical and deep white matter signal hyperintensity with high signal in the splenium of the corpus callosum and the globus pallidus. There was no enhancement with gadolinium. Vitamin E level, arylsulphatase A activity, and very long chain fatty acids were normal. Lymphocyte mitochondrial DNA studies (studies performed at the Children's Hospital of Los Angeles) showed missense substitutions at $\mathrm{mt15257}$ ( $\mathrm{G}$ to $\mathrm{A}$, aspartate to asparagine in cytochrome $b$ subunit of complex III) and $\mathrm{mt} 4216$ ( $\mathrm{T}$ to $\mathrm{C}$, tyrosine to histidine in ND1 subunit of complex I).

\section{Discussion}

These mitochondrial missense mutations are associated strongly with Leber's hereditary optic neuropathy (LHON) and studies of LHON pedigrees have disclosed a variety of neurological and non-neurological deficits. ${ }^{12}$ Short stature, ataxia, hyperreflexia, dorsal column deficits, acute encephalopathy, variable progression of findings, involuntary movements, dysarthria, and central demyelination have all been noted with mitochondrial mutations associated with $\mathrm{LHON}^{1-4}$ In addition, this subject had sensorineural hearing loss, diabetes, and cardiac conduction deficits, all findings seen in mitochondrial DNA disorders. Her family history, while sketchy, is consistent with maternal inheritance.

Corneal dystrophy has not been associated previously with LHON or any other mitochondrial disorder and is it possible that this subject suffered from two rare but independent disorders. Several facts about Fuch's dystrophy are consistent with a causative role for mitochon- drial defects. Postmitotic cells with high energy demands are particularly susceptible to mitochondrial defects. In Fuch's dystrophy, the primary defect is thought to be corneal endothelial cell dysfunction. Corneal endothelial cells are terminally differentiated and, because of their role in maintaining ion gradients for corneal fluid homeostasis, probably possess high aerobic metabolism requirements. Studies of mitochondrial enzymes are consistent with mitochondrial defects in Fuch's dystrophy. ${ }^{56}$ Fuch's dystrophy is known to be inherited, though a simple mendelian pattern of inheritance has not been found. ${ }^{7}$

The findings in this subject and the biology of corneal endothelial cells suggest that mitochondrial defects are involved in the pathogenesis of Fuch's endothelial dystrophy. This hypothesis can be tested in several ways. Pedigrees of Fuch's endothelial dystrophy victims can be scrutinised for evidence of maternal inheritence. As keratoplasty is the usual therapy for Fuch's endothelial dystrophy, endothelium from surgically extracted corneas can be evaluated for mitochondrial genetic defects or other evidence of mitochondrial dysfunction.

1 Shoffner JM, Wallace DC. Oxidative phosphorylation diseases. In: Scriver C, Beaudet A, Sly W, Valle D, eds. The metabolic and molecular bases of inherited diseases. 7 th ed. metabolic and molecular bases of

2 Wallace DC. A new manifestation of Leber's disease and a new explanation of the agency responsible for its unusual pattern of inheritence. Brain 1970;93:121-32.

3 Harding AE, Sweeney MG, Muller DH, et al. Occurrence of a multiple sclerosis-like illness in women who have a Leber's hereditary optic neuropathy mitochondrial DNA mutation. Brain 1992;115:979-89.

4 Flanigan KM, Johns DR. Association of the 11778 mitochondrial DNA mutation and demyelinating disease. Neurology 1993;43:2720-2.

5 McCartney MD, Robertson DP, Wood TO, McLaughlin BJ. ATPase pump site density in human dysfunctional corneal endothelium. Invest Opthalmol Vis Sci 1987;28:1955-62.

6 Tuberville AW, Wood TO, McLaughlin BJ. Cytochrome Tuberville AW, Wood TO, McLaughlin BJ. Cytochrome
oxidase activity of Fuch's endothelial dystrophy. Curr Eye Res 1986;5:939-47

7 Magovern M, Beauchamp GR, McTigue JW, Fine BS, Baumiller RC. Inheritence of Fuch's combined dystrophy. Ophthalmology 1979;86:1987-23. 